\begin{tabular}{|c|c|c|}
\hline & $\begin{array}{c}\text { European Association for the } \\
\text { Development of Renewable Energies, Environment } \\
\text { and Power Quality (EA4EPQ) }\end{array}$ & $\begin{array}{l}\text { International Conference on Renewable Energies and Power Quality } \\
\text { (ICREPQ'12) } \\
\text { Santiago de Compostela (Spain), 28th to 30th March, } 2012\end{array}$ \\
\hline
\end{tabular}

\title{
Energy Storage for Active Network Management on Electricity Distribution Networks with Wind Power
}

\author{
Stephen Carr ${ }^{1}$, Giuliano C. Premier ${ }^{2}$, Richard M. Dinsdale ${ }^{1}$, Alan J. Guwy ${ }^{1}$, and Jon Maddy ${ }^{1}$ \\ ${ }^{1}$ Sustainable Environment Research Centre (SERC) \\ Faculty of Health, Sport and Science, University of Glamorgan \\ Pontypridd, Mid-Glamorgan, CF37 1DL, United Kingdom \\ Phone number: +0044 1639 816426, e-mail: sjwcarr@glam.ac.uk \\ ${ }^{2}$ Sustainable Environment Research Centre (SERC) \\ Faculty of Advanced Technology, University of Glamorgan \\ Pontypridd, Mid-Glamorgan, CF37 1DL, United Kingdom
}

\begin{abstract}
Increasing amounts of variable renewable generation are likely to enter our energy systems in the near future. In order to accommodate this generation onto our electricity networks, the concept of active network management (ANM) has become a significant area of research interest. Network connected energy storage is considered here as a means to actively control the network in order to increase the amount of generation it is possible to connect to a network. Energy storage is one of several potential methods of ANM, but has not been widely researched in this context. In this paper the ability of energy storage to increase the amount of wind energy accepted onto a network is assessed over a range of round trip storage efficiencies. The results show that energy storage is able to increase the energy accepted onto a distribution network, with the efficiency of the energy storage, energy storage capacity, wind farm size, network losses and network characteristics being important in determining the relative effectiveness of energy storage.
\end{abstract}

\section{Key words}

Energy storage, Active network management, Optimal power flow, Wind power, Modelling and simulation

\section{Introduction}

Renewable energy is expected to make an increasing contribution to UK and worldwide energy supplies in the near future [1]. Much of this renewable energy is likely to be from variable sources, of which wind power is currently the most significant. The power level at which this will be delivered often makes it most suitable for connection to the electricity distribution network. Connection of an increasing amount of intermittent renewable energy onto electricity distribution networks requires new methods by which to manage and operate these networks [2].

A number of studies have addressed the issue of maximising the amount of generation it is possible to connect to a distribution network, with an increasing amount of interest in an active approach to tackling the problem [3]. Active network management (ANM) seeks to increase the amount of energy accepted from the generators attached to a network by adjusting network or generator parameters without upgrading the network infrastructure. To analyse networks in this way, techniques such as optimal power flow (OPF) have been used. Several studies focusing on the use of power curtailment, reactive power control and on load tap changing (OLTC) transformer control in order to assess the potential of active management schemes to aid in the integration of wind power and other forms of distributed generation onto distribution networks, have been reported previously[4]-[7]. AC OPF has also been combined with time-series to maximise the energy accepted onto a network from a variety of different renewable resources including wind, tidal and wave generation, utilising energy curtailment to maintain network parameters within limits [8].

Energy storage can be considered to be a potential component of active management schemes on electricity distribution networks [9], however its use has not been investigated in previous studies such as those listed above. Studies considering energy storage with wind power to date, have considered the operation of a single wind farm and modelled the distribution network constraints as a constant limit on the amount of energy which can be exported $[10,11]$, or based constraints on the voltage rise at that point [12].

This paper considers for the first time, the application of OPF to electricity distribution networks operating with multiple wind farms and energy storage. The operation of storage is included in the objective function of the OPF, and considers the efficiency of the storage. Time-series $\mathrm{AC} \mathrm{OPF}$ analyses are run for a number of different scenarios. The formulation used for the OPF is presented, 
then the paper outlines the case studies used and scenarios analysed and the results obtained from using storage as an ANM technique.

\section{Problem Formulation}

The optimisation problem and objective function seeks to optimise the system as a whole. It hypothetically treats the distribution network, wind farms and storage facility as if they were owned and operated by one entity. The goal of this study is to maximise the utilisation of available wind power. By considering all components as one commercial entity having the single mutual goal of maximising wind energy utilisation, the question of the suitability of energy storage for relieving network congestion and maximising resource utilisation can be addressed.

\section{A. Optimal power flow}

The ability of the network to accept generation is evaluated using an OPF programme. Objective functions which maximise distributed generation, along with the use of OPF for this purpose has been demonstrated previously [4, 5, 13]. In this paper an objective function which maximises energy accepted from wind power generators on a network including storage is developed and demonstrated. The OPF routine used in this study is adapted from that in MATPOWER [14], and is modified to make it suitable for time series analysis of distribution networks with wind farms and energy storage. To model wind farms, constant power factor generators are included by adding the power factor of the generator as a linear equality constraint. Energy storage is included by adding storage charging, discharging and capacity modules to the OPF formulation. The operation of OLTC transformers is included by adding the tap settings of the transformers to the control variables and updating the Jacobian of the nonlinear constraints.

MATPOWER runs in MatLAB ${ }^{\mathrm{TM}}$ and in this case

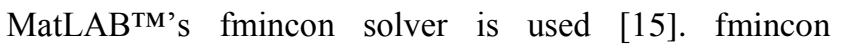
attempts to find the minimum of a constrained nonlinear multivariable function, in which class AC OPF is included. In order to analyse the performance of a system over the course of a year, time series of system load and wind power output are obtained, and an OPF carried out at each half hour time step over the course of a year. When analysing storage, this time series approach is necessary in order to effectively capture the process of the store filling and emptying.

\section{B. Objective Functions}

When maximising the renewable energy generation without storage on the network, the objective function used is given in equation (1). The maximum value of power $P_{g}$ for a given time period is a function of the capacity of the generator and the magnitude of the wind power time series for that time period. Equation (1) then maximizes the output of the generators over the given time period.

$$
\max \sum_{g \in G} P_{g}
$$

Where $P_{g}$ is the power output of wind power generator $g$ out of the set of wind power generators $G$.

When maximising energy delivered to the network with storage, objective function (2) is used:

$$
\max \left(\sum_{g \in G} P_{g}-\left(1-e_{r t}\right) \sum_{s \in S} P_{s}\right)
$$

Where $P_{S}$ is the power taken in by storage module $S$ out of the set of storage modules $S$, and $e_{r t}$ is the round trip efficiency of the storage modules. This objective function maximises the difference between the extra energy from the generator entering the network over a time period and the energy which will be lost due to the round trip efficiency of the storage.

Equation (2) is only used as an objective function when it would be necessary to curtail wind power if there were no storage on the network. At other times the wind power output is held at the full available output and the objective function maximises the amount of energy exiting storage back to the grid. In order to determine whether curtailment occurs, and so which objective function to use, an OPF routine is executed, initially without storage on the network, using equation (1) as the objective function.

\section{Constraints}

The constraints take into account the real and reactive power flows at each bus as well as the thermal, voltage, transformer and generator limits [3].

Additional constraints are needed when considering energy storage in the OPF. When optimising with energy storage, constraint:

$L_{S}(t+1)=L_{S}(t)+\sqrt{e_{r t}} P_{S}^{i n}(t)-\left(\frac{1}{\sqrt{e_{r t}}}\right) P_{S}^{\text {out }}(t)$

is used.

If there is curtailment:

Or if there is no curtailment:

$$
P_{S}^{\text {out }}(t)=0
$$

$$
P_{S}^{\text {in }}(t)=0
$$

$$
P_{g, i}(t)=P_{g, i}^{\max }(t)
$$

where $L_{S}$ is the fill level of the storage facility; $P_{S}^{\text {in }}$ is the power entering storage and $P_{S}^{\text {out }}$ is the power exiting storage.

Equation (3) ensures that the storage level $L_{s}$ at the subsequent time step accounts for the energy entering or leaving the storage during the current time step. Equations (4) and (5) ensure that the storage facilities cannot input and output power at the same time. Equation 
(6) ensures that the OPF does not result in extra wind power being curtailed to allow extra output power from the storage. The generators operate at a power factor of unity.

\section{Case Study}

A distribution network obtained from the UK generic distribution system website (UKGDS) [16] is used as a case study. The network represents a simplified radial distribution network. It was developed by UKGDS to analyse active network management techniques. It is a 33 $\mathrm{kV}$ network supplying 38.94 MVA of load at a power factor of 0.98 . The network is mostly radial in nature. A diagram of the network is shown in Figure 1.

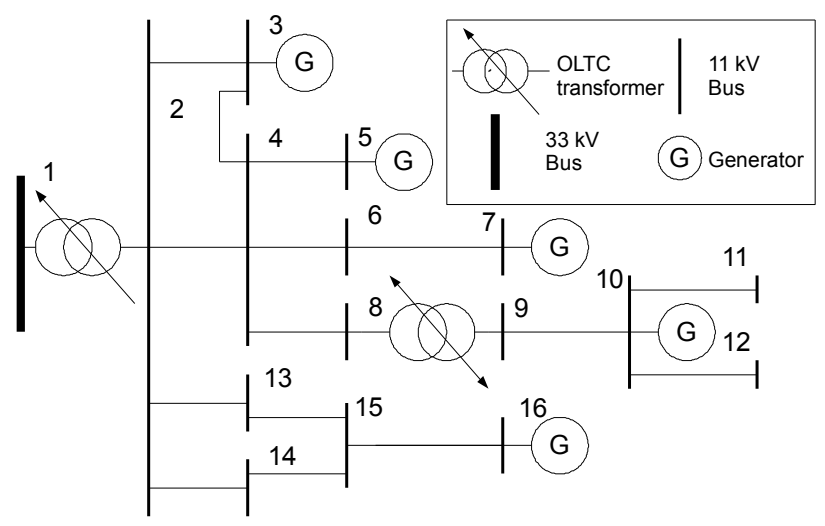

Fig. 1. Diagram of Network: A generic radial distribution network

\section{A. Capacity analyses and determination of nodal capacities}

Five wind farm sites are chosen to be at locations remote from the main grid connection point and are generally located at the ends of individual feeders in order to represent plausible locations for wind farms. Three different wind farm penetration levels are considered:

a) The wind farms are sized such that at minimum load they have the maximum combined capacity with no power needing to be curtailed, representing a 'fit and forget' approach. This method reflects the current (passive) methodology of allocating generating capacity, but does not take constraints such as fault levels, substation reverse power flow and N-1 security constraints, whereby the system can withstand the loss of any single component, into account.

b) The wind farms are sized such that, considered individually their maximum output can be accepted at maximum load on the network. This sizing gives wind farm capacities which are suitable for each individual node, but cause curtailment when considered together.

c) Each wind farm bus has a capacity of $150 \%$ of that determined in b). This will increase the level of curtailment experienced, allowing a comparison of the effectiveness of energy storage at different capacity levels.

The capacity allocation is carried out with generators operating at unity power factor. Table I presents the wind farm capacity allocations for each scenario [22].

Table I- Wind farm capacity allocation scenarios

\begin{tabular}{c|ccc}
\hline $\begin{array}{c}\text { Wind farm } \\
\text { bus }\end{array}$ & \multicolumn{3}{|c}{ Capacity (MW) } \\
\hline & $a)$ & $b)$ & $c)$ \\
3 & 12.82 & 28.76 & 43.14 \\
5 & 15.24 & 51.69 & 77.54 \\
7 & 1.34 & 10.84 & 16.26 \\
10 & 2.63 & 14.4 & 21.6 \\
16 & 3.04 & 3.34 & 5.01 \\
\hline Total & 35.07 & 109.03 & 163.55 \\
\hline
\end{tabular}

The case of centralised storage is investigated in this paper, whereby the network only has one storage facility associated with it and the storage bus is chosen such that it has the greatest benefit in terms of allowing extra energy onto the network. This location is determined by running an OPF at both maximum and minimum load on the network. For this network both bus 5 and bus 10 are analysed individually [17], as they both have very similar benefits. Other potential scenarios include storage at all wind farm busses, or storage distributed amongst the load.

\section{A. Time series}

The network is analysed over a yearly time frame by using both load and wind farm output time series in combination with an OPF analysis. By using a time series analysis, the many different combinations of load and wind power output are included, and the behaviour of the storage over the course of a year can be assessed. The time series define the load on the system and the wind farm output at half hourly periods over the course of a year. The optimum amount of energy accepted by the network from the wind farm over the course of a year can be determined by applying an OPF analysis at each half hourly time period.

Time series for the wind farm output and load at half hourly intervals are obtained from the UK Generic Distribution System (UKGDS) at SEDG [16]. The load is based on a mix of domestic, industrial and commercial load. The values of the loads and wind farm outputs are then adjusted from their maximum outputs by the factor given in the time series.

\section{B. Curtailment analysis}

The network is first analysed with no storage. This gives a base case against which energy storage can be compared. The results are shown in Table II. It can be seen that by increasing the capacity and allowing curtailment, a large amount of extra energy can be accepted onto the network. However, this is achieved with an increasing proportion of energy available from the wind farms being curtailed, which could increase the 
cost at which the electricity is produced, reducing its financial viability. Only $22 \%$ of the extra energy available through sizing wind farms at penetration level $c$ compared to penetration level $b$ is accepted onto the network, demonstrating the increased congestion with increasing wind farm size.

Table II.- Network capacities and wind energy accepted or

\begin{tabular}{cccc}
\multicolumn{4}{c}{ curtailed by network } \\
\hline $\begin{array}{c}\text { Capacity } \\
\text { MW }\end{array}$ & $\begin{array}{c}\text { Potential } \\
\text { Output } \\
\text { GWh }\end{array}$ & $\begin{array}{c}\text { Accepted } \\
\text { GWh(\%) }\end{array}$ & $\begin{array}{c}\text { Curtailed } \\
\text { GWh }(\%)\end{array}$ \\
\hline 35.07 & 83.3 & $83.3(100)$ & 0 \\
109.0 & 259.0 & $184.4(71.2)$ & $74.6(28.8)$ \\
163.5 & 388.5 & $213.2(54.8)$ & $175.4(45.2)$ \\
\hline
\end{tabular}

\section{Storage Analysis}

The following analysis is carried out with the charging and discharging capacity unconstrained, whilst the storage capacity is assessed at three different levels. These capacities are determined by the number of equivalent hours of full wind farm output. The three levels are classified as short term (1 hour), medium term (10 hours) and long term (100 hours). Unconstrained storage is also assessed. With the storage capacity unconstrained, unrealistically large capacities can be required (i.e. greater than 1000 hours for some scenarios), especially at large efficiencies. Constraining the storage capacities may result in an increased amount of energy being curtailed. It is assumed that any energy remaining in storage at the end of the analysis period will be discharged to the network at some future point at the given discharge efficiency.
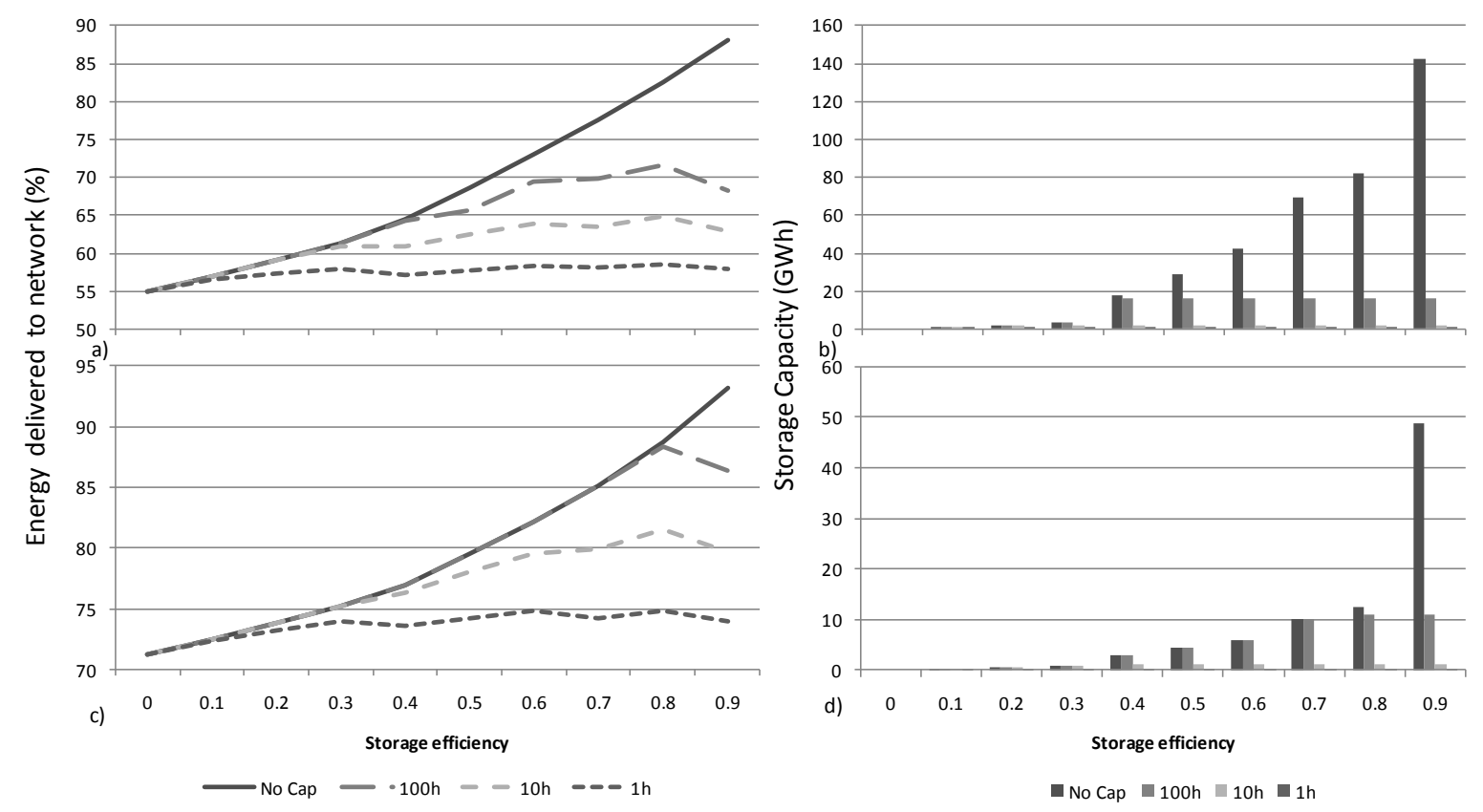

Fig. 2. a) Energy delivered to network with penetration level $c$, b) storage capacity required with penetration level $c$, c) Energy delivered to network with penetration level $b, \mathrm{~d}$ ) storage capacity required with penetration level $b$ for Network 1 with storage located at bus 5
Figure 2 shows the overall energy delivered to the network with storage located at bus 5 , along with the storage capacity required to achieve this. Figure 3 shows this for storage located at bus 10 . With the storage capacity unconstrained, increasing efficiency will always lead to increasing amounts of energy being delivered to the network, however very large storage capacities can be required, which may be technologically and economically unfeasible. In some cases energy can build up over the course of the year, resulting in a large proportion of the available energy (i.e. up to $38 \%$ ) remaining in the store at the end of the year. With the storage capacity constrained, a reduction in the energy delivered can occur. A storage capacity of $1 \mathrm{~h}$ results in small increases in energy delivered for all efficiencies. Storage capacities of $10 \mathrm{~h}$ and $100 \mathrm{~h}$ can both result in significant increases in energy delivered. The costs of the technologies required, and the amount of extra energy delivered will be important in determining the feasibility of energy storage, and the additional benefit obtained from the storage.

At penetration level $c$ a greater proportion of the energy is available for the store, so greater gains can be made by the storage. Component requirements are generally larger, so the economics will not necessarily be better. Increased wind farm capacity means energy is curtailed more frequently, meaning that there is less opportunity for the store to discharge, exacerbating problems with energy accumulating in the storage. In this case, using an energy storage mechanism such as hydrogen, which allows the stored energy to be used for other purposes, may be suitable. 

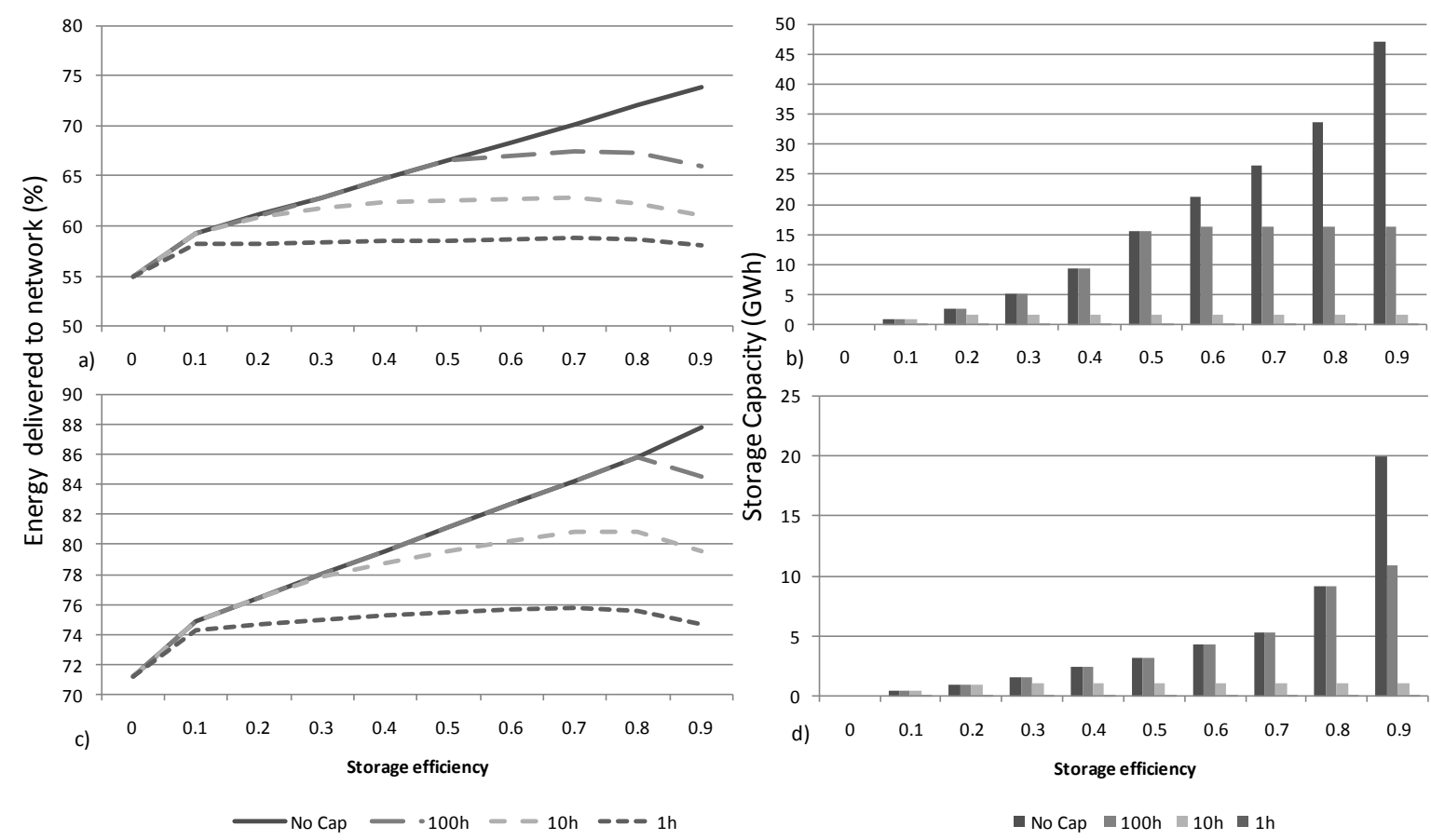

Fig. 3. a) Energy delivered to network with penetration level $c$, b) storage capacity required with penetration level $c$, c) Energy delivered to network with penetration level $b, \mathrm{~d}$ ) storage capacity required with penetration level $b$ for with storage located at bus 10

The charge and discharge capacities obtained in the analysis are not shown in Figure 2 and Figure 3. The maximum charging capacity varies with efficiency as shown in Figure 4, for penetration level $b$ with storage unconstrained and located at bus 5. At round trip efficiencies of $10-30 \%$, the maximum charging capacity required is $35.8 \mathrm{MW}$. This increases to $73.0 \mathrm{MW}$ at efficiencies of $40-80 \%$ and $84.5 \mathrm{MW}$ at $90 \%$ efficiency. This can reduce slightly when storage capacity is constrained. This shows it is important to take the storage efficiency into account in the OPF formulation when analysing networks in this way.

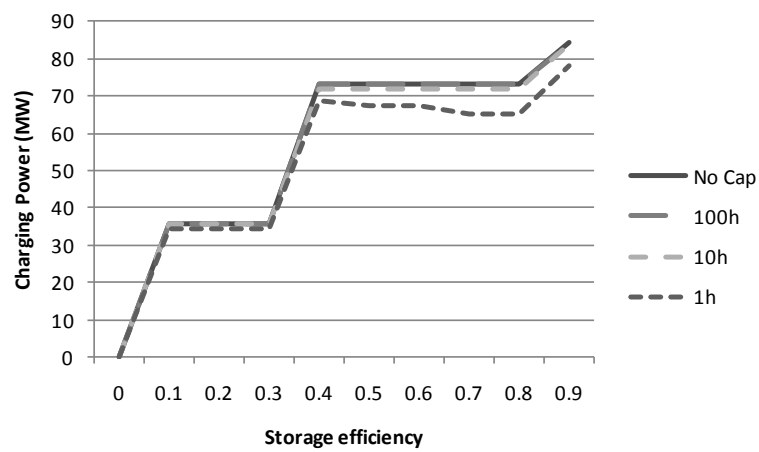

Fig. 4. Variation in maximum charging power with efficiency for different storage capacities. Penetration level $b$ with storage located at bus 5

When the storage capacity is constrained, the gain in extra energy delivered with increasing efficiency can be small, and in some cases the extra energy delivered may reduce with increasing efficiency, as seen in Figure 2 and Figure 3. As the storage efficiency increases, the round trip efficiency loss from taking energy into the store decreases. With unconstrained capacity the amount of energy curtailed either remains the same or reduces and consequently the amount of energy delivered to the network increases. With a capacity constraint on the store, in some instances where the store reaches its capacity, an increase in curtailment with increasing efficiency due to the capacity constraint is not matched by the reduction in round trip losses caused by the increase in efficiency, resulting in an overall reduction in energy delivered to the network.

\section{Discussion}

Energy storage potentially adds extra value to the renewable energy resource by allowing the time of release of the energy to be controlled. In this analysis, energy is discharged from the storage facility at the earliest available opportunity. Energy storage could be used for more than one purpose, e.g. as well as allowing extra energy onto the network, value could be added to the electricity generated by controlling the time of release, e.g. in conjunction with price variations in an electricity spot market [18] in order to generate extra profits. In some markets such as the UK the majority of electricity is traded bilaterally with the option to trade on power exchanges. In this case it may be possible to increase the value of the stored electricity by using it to reduce costs in the electricity balancing market, supply peak demand, or reduce variation in output.

With centralised storage, the efficiency of the storage does affect the optimal amount of power for the storage to absorb. The range of efficiencies over which this variation occurs is typical of a number of storage technologies, i.e. hydrogen storage at $40 \%$ to battery/flywheel storage at efficiencies up to $90 \%$ [12], so is important to consider when operating such systems. 
This effect has been observed for other storage configuration scenarios (e.g. dispersed storage located at load busses), though the results are not presented in this paper.

\section{Conclusions}

An OPF formulation including energy storage has been developed and used to analyse the use of energy storage as an ANM component on electricity distribution networks with wind power. Including energy storage explicitly in an optimal power flow formulation allows the optimal capacity of components to be determined in order to maximise the energy delivered to the network. It can be seen that, depending on the system setup, the optimal component sizes vary with storage efficiency, so it is important to include the efficiency in the objective function.

Constraining the capacity of the store can result in less overall energy delivered to network, but prevents unrealistically large amounts of energy accruing in the store. The level to which the capacity is constrained has a large bearing on the benefit obtained from the storage. The amount of extra energy which storage delivers to the network is dependent on the storage location, wind farm size, storage efficiency and capacity constraint.

\section{Acknowledgement}

This work was supported in part by the UK- SUPERGEN SHEC EP/E040071/1 and GR/S26965/01 and by the CymruH2Wales project, part of the Low Carbon Research Institute Convergence Programme. The CymruH2Wales project has been supported by the European Development Fund through the Welsh Government.

\section{References}

[1] "UK energy white paper: meeting the energy challenge," BERR, 2007.

[2] "A Technical Review and Assessment of Active Network Management Infrastructures and Practices, Report to U.K. Department of Trade and Industry (DTI)," EA Technology, 2006.

[3] P. Djapic, C. Ramsay, D. Pudjianto, G. Strbac, J. Mutale, N. Jenkins, and R. Allan, "Taking an active approach," IEEE Power \& Energy Magazine, vol. 5, pp. 68-77, Jul-Aug 2007.

[4] S. N. Liew and G. Strbac, "Maximising penetration of wind generation in existing distribution networks," IEE Proceedings-Generation Transmission and Distribution, vol. 149, pp. 256-262, May 2002.
L. F. Ochoa, C. J. Dent, and G. P. Harrison, "Distribution network capacity assessment: variable DG and active networks," IEEE Transactions on Power Systems, vol. 25, pp. 87-95, Feb 2010.

[6] R. A. F. Currie, G. W. Ault, C. E. T. Foote, and J. R. McDonald, "Active power-flow management utilising operating margins for the increased connection of distributed generation," IET Generation Transmission \& Distribution, vol. 1, pp. 197-202, Jan 2007.

[7] P. N. Vovos, A. E. Kiprakis, A. R. Wallace, and G. P. Harrison, "Centralized and distributed voltage control: Impact on distributed generation penetration," IEEE Transactions on Power systems, vol. 22, pp. 476-483, Feb 2007.

[8] T. Boehme, G. P. Harrison, and A. R. Wallace, "Assessment of distribution network limits for nonfirm connection of renewable generation," IET Renewable Power Generation, vol. 4, pp. 64-74, Jan 2010.

[9] J. McDonald, "Adaptive intelligent power systems: Active distribution networks," Energy Policy, vol. 36, pp. 4346-4351, Dec 2008.

[10] M. Korpaas and T. Gjengedal, "Oppertunities for Hydrogen Storage in connection with Stochastic Distributed Generation," in 9th International Conference on Probabilistic Methods Applied to Power Systems, KTH, Stockholm Sweden, 2006.

[11] M. Korpas and C. J. Greiner, "Opportunities for hydrogen production in connection with wind power in weak grids," Renewable Energy, vol. 33, pp. 11991208, Jun 2008

[12] J. P. Barton and D. G. Infield, "Energy storage and its use with intermittent renewable energy," IEEE

Transactions on Energy Conversion, vol. 19, pp. 441448, Jun 2004

[13] G. P. Harrison and A. R. Wallace, "Optimal power flow evaluation of distribution network capacity for the connection of distributed generation," IEE Proceedings-Generation Transmission and Distribution, vol. 152, pp. 115-122, Jan 2005. http://www.pserc.cornell.edu/matpower/, "MATPOWER," 2006.

[15] Optimization Toolbox For use with Matlab: The Mathworks, Inc.

[16] http://www.sedg.ac.uk/, "UKGDS," 2007.

[17] S.J.W Carr "The integration of hydrogen generation with embedded renewable energy systems" $\mathrm{PhD}$ Thesis, University of Glamorgan, 2010

[18] M. Korpas and A. T. Holen, "Operation planning of hydrogen storage connected to wind power operating in a power market," IEEE Transactions on Energy Conversion, vol. 21, pp. 742-749, Sep 2006. 